\title{
The Connection Dependent Threshold Model for Finite Sources - A Generalization of the Engset Multirate Loss Model
}

\author{
Ioannis D. Moscholios and Michael D. Logothetis \\ University of Patras, Dept. of Electrical \& Computer Engineering, \\ Wire Communications Laboratory, 26500 Patras, Greece \\ \{moscholios, m-logo\} @wcl.ee.upatras.gr
}

\begin{abstract}
Call Blocking Probabilities (CBP) are the key index of the call-level QoS in multirate networks supporting either CBR or elastic traffic. We review the Engset Multirate Loss Model, in a single link, for CBR traffic, and for elastic traffic we propose the Connection Dependent Threshold Model with finite sources (f-CDTM). In f-CDTM, calls may adjust their traffic and bandwidth requirements according to sets of thresholds. Furthermore, we present the fCDTM for a mixture of service-classes of finite and infinite sources. The proposed models don't have a product form solution; therefore the CBP calculation is based on approximate but recursive formulas used for the link occupancy distribution determination. The latter is complex since it requires enumeration and processing of the system state space. Simulation results validate our analysis.
\end{abstract}

\section{Introduction}

The classical Erlang Multirate Loss Model (EMLM) analyzes the call blocking behavior of service-classes with different bandwidth-per-call requirements when they are accommodated in a single link loss system. Calls of each service-class arrive to the link according to a Poisson process and compete for the available link bandwidth under the Complete Sharing (CS) policy. If the required bandwidth is available, calls are accepted and remain in the system for an arbitrarily distributed service time [1]; if not, calls are blocked and lost. The fact, that the EMLM is described by an efficient recursive formula ([1],[2]), not only simplifies the determination of Call Blocking Probability (CBP), but also serves as the basis in the analysis of other loss models [3][5].

In [3], single and multi retry models are proposed, in which blocked calls can retry (one or more times) to be connected in the system with reduced bandwidth and increased service time requirements. In [4], Single and Multi Threshold Models are proposed, in which the bandwidth requirement of a new call may depend on one or more thresholds, which indicate the occupied link bandwidth, $j$. In [5] the retry and threshold models, as well as the EMLM, are generalized to the Connection Dependent Threshold Model (CDTM); a threshold model, in which the state dependency is individualized among service-classes. In [6] the EMLM is extended to the Engset Multirate Loss Model (EnMLM) where the offered traffic of each service-class $k$, comes 
from a finite number of $N_{k}$ sources. In EnMLM the CBP calculation is based on a recursive formula, similar to the EMLM. However, the determination of the link occupancy distribution, $G(j)$, which is essential for the CBP calculation, is complex. This is because the system state space needs enumeration and processing (in order for an equivalent system to be defined) prior to the $G(j)$ calculation.

In this paper, the EnMLM is extended to the CDTM for finite sources (f-CDTM), where each service-class has its own set of thresholds and calls may reduce their bandwidth and increase their service time requirements according to the value of $j$ and the thresholds. We generalize the f-CDTM to include a mixture of service-classes with either finite or infinite sources. In the proposed models the determination of $G(j)$, is complex, since the state space requires enumeration and processing. We evaluate the accuracy of the proposed models by comparing analytical with simulation CBP results. The comparison is based on the Relative Approximation Errors (RAE); as a reference point, we use the RAE of the corresponding infinite source models.

In section 2 we propose the f-CDTM. We present the analytical model and prove the recursive formula used for the $G(j)$ calculation (not for the general case, due to lack of space). We proceed to the generalization to the CDTM to include a mixture of service-classes of either finite or infinite number of sources. In section 3 we present application examples. We conclude in section 4.

\section{The Proposed CDTM for Finite Sources (f-CDTM)}

A call of a service-class $k$ is accepted in the system with its requirements $\left(\mathrm{b}_{\mathrm{kc}_{\mathrm{t}}}, \mu_{\mathrm{kc}_{\mathrm{t}}}{ }^{-1}\right), t$ $=1, \ldots, T(k)$, when $J_{k_{t-1}}<j \leq J_{k_{t}}$, where $J_{T(k)}=C-b_{k c_{T(k)}}$ and $T(k)$ is the number of thresholds $(J)$ and of contingency bandwidth requirements of call k (Fig. 1).

Eq. (1) is proposed for the $G(j)$ 's calculation.

$$
G(j)=\left(\begin{array}{c}
1 \text { for } j=0 \\
\frac{1}{j} \sum_{k=1}^{K}\left(N_{k}-n_{k}+1\right) \alpha_{k} b_{k} \delta_{k}(j) G\left(j-b_{k}\right)+ \\
+\frac{1}{j} \sum_{k=1}^{K} \sum_{t=1}^{T}\left(N_{k}-\left(n_{k}+n_{k c_{1}}+\ldots+n_{k c_{t}}+\ldots+n_{k c_{T}}\right)+1\right) a_{k c_{t}} b_{k c_{t}} \delta_{k c_{t}}(j) G\left(j-b_{k c_{t}}\right) \text { for } j=1, \ldots, C \\
0 \quad \text { otherwise }
\end{array}\right\rangle
$$

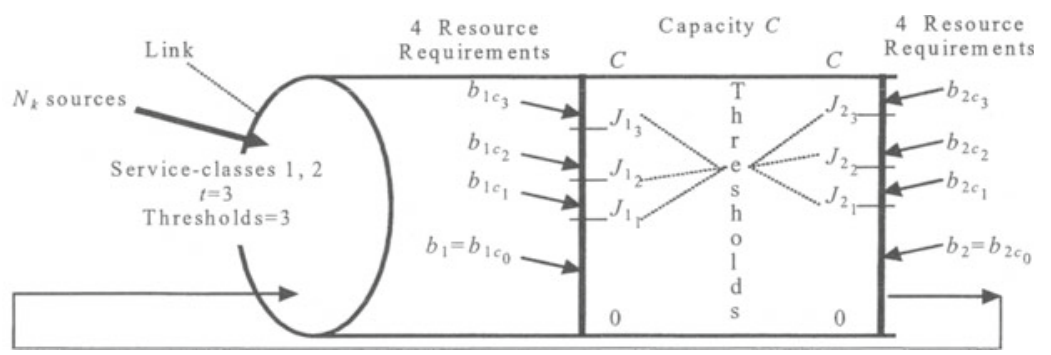

Fig. 1. Principle of the f-CDTM 
- $\delta_{k}(\mathrm{j})=1$ when $1 \leq j \leq C$ and $b_{k c}=0$, or, when $j \leq J_{k_{t}}+b_{k}$ and $b_{k c}>0$, otherwise $\delta_{k}(j)=0$.

- $\delta_{k_{c_{t}}}(\mathrm{j})=1$ when $J_{k_{t}}+b_{k c_{t}} \geq \mathrm{j}>J_{k_{t-1}}+b_{k c_{t}}$, otherwise $\delta_{k c_{t}}(j)=0$.

The CBP, $B_{k c_{T(k)}}$, that a call $\mathrm{k}$ is blocked with its last bandwidth requirement, $b_{k c_{T(k)}}$, is defined as $B_{k c_{T(k)}}=\operatorname{Prob}\left\{j>b_{k c_{T(k)}}\right\}$ and is given by:

$$
B_{k c_{T(k)}}=\sum_{j=C-b_{k c}(k)}^{C} G^{-1} G(j) \text { where } G=\sum_{j=1}^{C} G(j)
$$

To simplify the proof of (1), let us consider two service-classes only with the same single threshold, $J_{0}$, and bandwidth and service-time requirements $\left(b_{1}, b_{2}\right),\left(\mu_{1}^{-1}, \mu_{2}^{-1}\right)$, respectively. Calls of the $1^{\text {st }}$ service-class use the pair $\left(b_{1 c}, \mu_{1 c}^{-1}\right)$ when $j>J_{0}$. Although this model does not have a PFS, we assume that Local Balance (LB) equations are valid for calls of the $1^{\text {st }}$ service-class:

$$
\begin{gathered}
{\left[N_{1}-n_{1}+1\right] a_{1} b_{1} G\left(j-b_{1}\right)=E\left(n_{1} b_{1} \mid j\right) G(j) \text { for } j-b_{1} \leq J_{o}} \\
{\left[N_{1}-\left(n_{1}+n_{1 c}\right)+1\right] a_{1 c} b_{1 c} G\left(j-b_{1 c}\right)=E\left(n_{1 c} b_{1 c} \mid j\right) G(j) \text { for } j-b_{1 c}>J_{o}}
\end{gathered}
$$

where: $\alpha_{1}, \alpha_{1 \mathrm{c}}$ are the offered traffic loads per idle source of the $1^{\text {st }}$ service-class, such that $\alpha_{1} b_{1}=\alpha_{1 \mathrm{c}} b_{1 \mathrm{c}}$, and $n_{1 \mathrm{c}}$ is the number of in-service $1^{\text {st }}$ service-class sources (calls) accepted in the system with $b_{1 \mathrm{c}}$.

As far as calls of the $2^{\text {nd }}$ service-class are concerned, the L.B equation is of the form:

$$
\left[N_{2}-n_{2}+1\right] a_{2} b_{2} G\left(j-b_{2}\right)=E\left(n_{2} b_{2} \mid j\right) G(j) \text { for } 1 \leq j \leq C
$$

Equations (2) to (4) lead to a system of equations (6) to (8):

$$
\begin{aligned}
& {\left[N_{1}-n_{1}+1\right] a_{1} b_{1} G\left(j-b_{1}\right)+\left[N_{2}-n_{2}+1\right] a_{2} b_{2} G\left(j-b_{2}\right)=E\left(n_{1} b_{1}+n_{2} b_{2} \mid j\right) G(j) \text { for } 1 \leq j \leq J_{0}+b_{1 c}} \\
& {\left[N_{1}-n_{1}+1\right] a_{1} b_{1} G\left(j-b_{1}\right)+\left[N_{2}-n_{2}+1\right] a_{2} b_{2} G\left(j-b_{2}\right)+\left[N_{1}-\left(n_{1}+n_{1 c}\right)+1\right] a_{1 c} b_{c} G\left(j-b_{1 c}\right)=j G(j) \text { for } J_{0}+b_{1 c}<j \leq J_{0}+b_{1}} \\
& {\left[N_{2}-n_{2}+1\right] a_{2} b_{2} G\left(j-b_{2}\right)+\left[N_{1}-\left(n_{1}+n_{1 c}\right)+1\right] a_{1 c} b_{1 c} G\left(j-b_{1 c}\right)=E\left(n_{2} b_{2}+n_{1 c} b_{1 c}\right) G(j) \text { for } J_{0}+b_{1}<j \leq C}
\end{aligned}
$$

Eqs. (6)-(8) are combined into one for $G(j)$ 's calculation, under two approximations:

- Migration approximation (M.A) in eq. (6): $E\left(n_{1 c} b_{1 c} \mid j\right)$ is negligible in the region $1 \leq j \leq J_{0}+b_{1 c}$, i.e. calls of the $1^{\text {st }}$ service-class accepted with $b_{1 c}$ are assumed to be negligible when $1 \leq j \leq J_{0}+b_{1 c}$.

- Upward migration approximation (UA) in eq. (8): $E\left(n_{1} b_{1} \mid j\right)$ is negligible in the region $J_{0}+b_{1}<j \leq C$, i.e. calls of the $1^{\text {st }}$ service-class, accepted with $b_{1}$, are negligible when $J_{0}+b_{1}<j \leq C$. The UA induces high RAE in the CBP results (section 3). Based on the M.A and U.A the calculation of $G(j)$ 's is given by:

$$
\begin{aligned}
& {\left[N_{1}-n_{1}+1\right] a_{1} b_{1} \delta_{1}(j) G\left(j-b_{1}\right)+\left[N_{2}-n_{2}+1\right] a_{2} b_{2} G\left(j-b_{2}\right)+} \\
& {\left[N_{1}-\left(n_{1}+n_{1 c}\right)+1\right] a_{1 c} b_{1 c} \delta_{1 c}(j) G\left(j-b_{1 c}\right)=j G(j)}
\end{aligned} \quad \text { for } 1<j \leq C
$$


$\delta_{2}(j)=1$ for $1 \leq j \leq J_{0}+b_{1}$ otherwise $\delta_{2}(j)=0, \delta_{2 c}(j)=1$ for $j>J_{0}+b_{1 c}$ otherwise $\delta_{2 c}(j)=0$.

Consider a single link that accommodates $K_{\text {fin }}$ service-classes of finite sources and $K_{\text {inf }}$ service-classes of infinite sources. Then, the calculation of $G(j)$ 's is as follows

$$
G(j)=\left\{\begin{array}{c}
1 \text { for } j=0 \\
\frac{1}{j} \sum_{k=1}^{K}{ }_{f i n}\left(N_{k}-n_{k}+1\right) \alpha k b k \delta_{k}(j) G\left(j-b_{k}\right)+ \\
+\frac{1}{j} \sum_{\mathrm{k}=1}^{K_{f i n}} \sum_{t=1}^{T(k)}\left(N_{k}-\left(n_{k}+n_{k c_{1}}+\ldots+n_{k c_{t}}+\ldots+n_{k c_{T}}\right)+1\right) a_{k c_{t} b_{k c_{t}} \delta_{k c_{t}}(j) G\left(j-b_{k c_{t}}\right)} \\
+\frac{1}{j} \sum_{k=1}^{K_{\text {inf }}} \alpha_{k} b_{k} \delta_{k}(j) G\left(j-b_{k}\right)+\frac{1}{j} \sum_{\mathrm{k}=1}^{K_{\text {inf }}} \sum_{t=1}^{T} a_{k c_{t}} b_{k c_{t}} \delta_{k c_{t}}(j) G\left(j-b_{k c_{t}}\right) \text { for } j=1, \ldots, C \\
0 \text { otherwise }
\end{array}\right\rangle
$$

- $\delta_{k}(\mathrm{j})=1$ when $1 \leq j \leq C$ and $b_{k c}=0$, or, when $j \leq J_{k_{t}}+b_{k}$ and $b_{k c}>0$, otherwise $\delta_{k}(j)=0$.

- $\delta_{k c_{t}}(\mathrm{j})=1$ when $J_{k_{t}}+b_{k c_{t}} \geq \mathrm{j}>J_{k_{t-1}}+b_{k c_{t}}$, otherwise $\delta_{k c_{t}}(j)=0$. This is the gener. f-CDTM.

\section{Numerical Example - Evaluation}

We present an application example to show the performance of the generalized $\mathrm{f}$ CDTM. We compare the CBP approximation errors that appear in the f-CDTM, with that of CDTM (with infinite population). The analytical CBP results are compared with simulation results (mean values of 7 runs with $95 \%$ confidence interval). In graphs (Fig. 2), we compare the CBP results obtained by the infinite and finite models by using the Mean Relative Approximation Error (MRAE) of CBP:

$$
M R A E=\frac{\sum_{k=1}^{K} R A E k}{K} 100 \%
$$

where $\mathrm{RAE}_{k}$ stands for the Relative Approximation Error for each service-class $k$ :

$$
R A E_{k}=\frac{\left|B_{k, a n}-B_{k, s i m}\right|}{B_{k, \text { sim }}}
$$

where $B_{k, a n}, B_{k, s i m}$ are CBP obtained by analytical models and simulation, respectively.

Consider a link of capacity $C=50$ b.u. and two service-classes which require $b_{1}=10$ and $b_{2}=7$ b.u., respectively. Calls of the $1^{\text {st }}$ service-class arrive to the link according to a Poisson process, whereas the offered traffic-load is $\alpha_{1}$. Calls of the $2^{\text {nd }}$ service-class arrive to the link according to a quasi-random process, whereas the offered trafficload per idle source is $\alpha_{2}$. Calls of the $1^{\text {st }}$ service-class use their reduced bandwidth requirement $b_{1 c_{1}}=8$ b.u when $j>J_{1_{0}}=30$, while $b_{1 c_{2}}=6$ b.u when $j>J_{1_{1}}=35$. In the first case the offered traffic load is given by $\alpha_{1 c_{1}}=\alpha_{1} b_{1} / b_{1 c_{1}}$, while in the second by $\alpha_{1 c_{2}}=$ $\alpha_{1} b_{1} / b_{1 c_{2}}$. Similarly, calls of the $2^{\text {nd }}$ service-class use their reduced bandwidth requirement $b_{2 c_{1}}=7$ b.u when $j>J_{1_{0}}=37$. In that case the offered traffic-load of calls of the sec- 
ond service-class is given by $\alpha_{2 c_{1}}=\alpha_{2} b_{2} / b_{2 c_{1}}$. Consider four values for the number of sources, $N_{2}$, of the $2^{\text {nd }}$ service-class. Table 1 shows the various values of $N_{2}$ and the corresponding values of $\alpha_{1}, \alpha_{2}, \alpha_{1 c_{1}}, \alpha_{1 c_{2}}, \alpha_{2 c_{1}}$. The equivalent system used for the CBP calculation is: $C=50019, b_{1}=10000, b_{2}=7001, b_{1 c_{1}}=8000, b_{1 c_{2}}=6000, b_{2 c_{1}}=4005, J_{1_{0}}=$ $30000, J_{1_{1}}=35000$ and $J_{2_{0}}=37001$. Fig. 2 shows the MRAE obtained from the generalized f-CDTM and the CDTM, for the four values of $N_{2}$. At each point in the horizontal axis entitled "offered traffic" $\alpha_{1}, \alpha_{1 c_{1}}, \alpha_{1 c_{2}}$ are constant, while $\alpha_{2}, \alpha_{2 c_{1}}$ are increased by $0.4 / N_{2}$ and $0.7 / N_{2}$ respectively, i.e. point 1 is $\left(\alpha_{1}, \alpha_{2}, \alpha_{1 c_{1}}, \alpha_{1 c_{2}}, \alpha_{2 c_{1}}\right)=\left(0.72,2.4 / N_{2}, 0.9\right.$, $\left.1.2,4.2 / N_{2}\right)$, point $2:\left(\alpha_{1}, \alpha_{2}, \alpha_{1 c_{1}}, \alpha_{1 c_{2}}, \alpha_{2 c_{1}}\right)=\left(0.72,2.8 / N_{2}, 0.9,1.2,4.9 / N_{2}\right), \ldots$, point 6: $\left(\alpha_{1}, \alpha_{2}, \alpha_{1 c_{1}}, \alpha_{1 c_{2}}, \alpha_{2 c_{1}}\right)=\left(0.72,4.4 / N_{2}, 0.9,1.2,7.7 / N_{2}\right)$. We present in Tables 2, 3, both analytical and simulation CBP results obtained from the generalized f-CDTM and the CDTM, for all points $(\mathrm{P}): 1, \ldots, 6$.

According to Fig. 2 higher MRAE appear when $N_{2}=12$. As the number of $N_{2}$ increases the values of the MRAE tend to be reduced, while they approach those of the CDTM. A similar behavior is observed in the case of the CBP results; the CBP are increased, approaching the CBP results in the CDTM case, when $N_{2}$ increases.

Table 1. Service-classes characteristics

\begin{tabular}{|c|c|c|c|c|c|c|}
\hline $\mathbf{N}_{\mathbf{k}}$ & Model used & $\boldsymbol{\alpha}_{\mathbf{1}}$ (erl) & $\boldsymbol{\alpha}_{\mathbf{2}}$ (erl) & $\boldsymbol{\alpha}_{\mathbf{1}_{\mathbf{1}}}$ (erl) & $\boldsymbol{\alpha}_{\mathbf{1 c}_{\mathbf{2}}}$ (erl) & $\mathbf{A}_{\mathbf{2 \mathbf { c } _ { 1 }}}$ (erl) \\
\hline$N_{1}=\infty, N_{2}=12$ & Gener.f-CDTM & 0.72 & $0.2\left(=2.4 / N_{2}\right)$ & 0.9 & 1.2 & $0.35\left(=4.2 / N_{2}\right)$ \\
\hline$N_{1}=\infty, N_{2}=60$ & Gener. f-CDTM & 0.72 & $0.04\left(=2.4 / N_{2}\right)$ & 0.9 & 1.2 & $0.07\left(=4.2 / N_{2}\right)$ \\
\hline$N_{1}=\infty, N_{2}=600$ & Gener. f-CDTM & 0.72 & $0.004\left(=2.4 / N_{2}\right)$ & 0.9 & 1.2 & $0.007\left(=4.2 / N_{2}\right)$ \\
\hline$N_{1}=N_{2}=\infty$ & CDTM & 0.72 & 2.4 & 0.9 & 1.2 & 4.2 \\
\hline
\end{tabular}

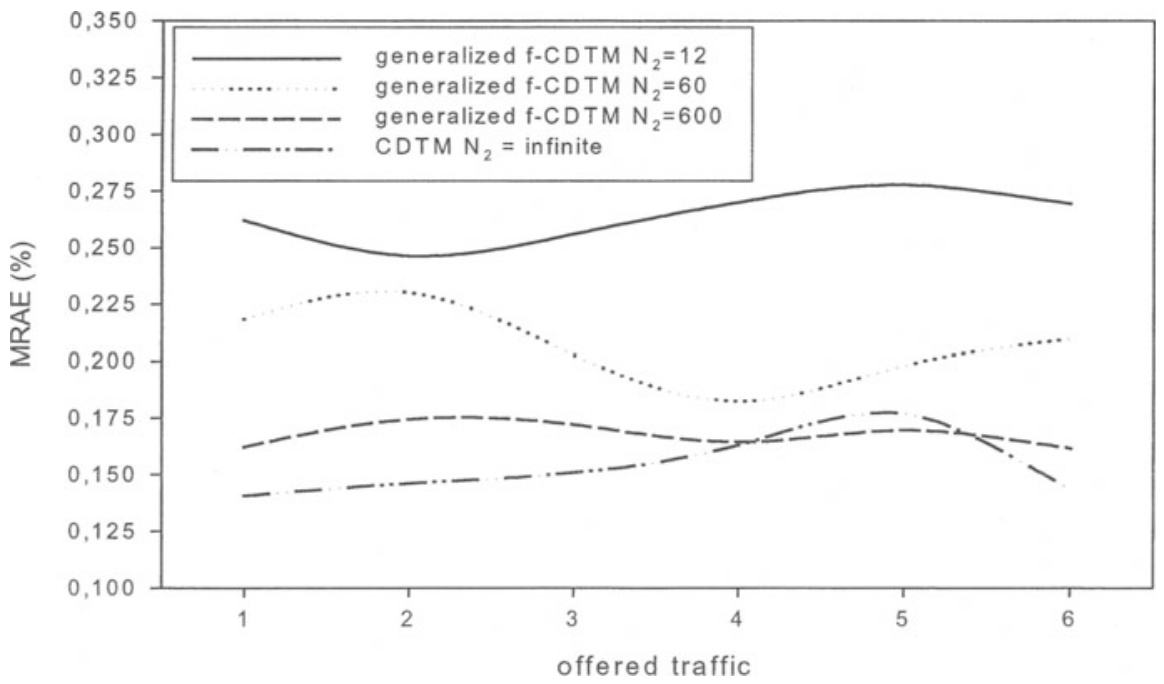

Fig. 2. MRAE of the generalized f-CDTM and the CDTM 
Table 2. Analytical results for the generalized f-CDTM and the CDTM

\begin{tabular}{|c|c|c|c|c|c|c|c|c|}
\hline & \multicolumn{2}{|c|}{$\begin{array}{c}N_{1}=\infty, N_{2}=12 \\
\text { (gener. f-CDTM) }\end{array}$} & \multicolumn{2}{c|}{$\begin{array}{c}N_{1}=\infty, N_{2}=60 \\
\text { (gener. f-CDTM) }\end{array}$} & \multicolumn{2}{c|}{$\begin{array}{c}N_{1}=\infty, N_{2}=600 \\
\text { (gener. f-CDTM) }\end{array}$} & \multicolumn{2}{|c|}{$\begin{array}{c}N_{1}=N_{2}=\infty \\
\text { (CDTM) }\end{array}$} \\
\hline $\mathrm{P}$ & $B_{1 c_{2}}(\%)$ & $B_{2 c_{1}}(\%)$ & $B_{1 c_{2}}(\%)$ & $B_{2 c_{1}}(\%)$ & $B_{1 c_{2}}(\%)$ & $B_{2 c_{1}}(\%)$ & $B_{1 c_{2}}(\%)$ & $B_{2 c_{1}}(\%)$ \\
\hline 1 & 2.31 & 1.31 & 3.92 & 2.18 & 4.43 & 2.45 & 4.49 & 2.48 \\
\hline 2 & 3.18 & 1.79 & 5.76 & 3.16 & 6.60 & 3.60 & 6.70 & 3.65 \\
\hline 3 & 4.20 & 2.35 & 8.03 & 4.36 & 9.26 & 5.01 & 9.39 & 5.10 \\
\hline 4 & 5.37 & 2.99 & 10.69 & 5.76 & 12.36 & 6.65 & 12.55 & 6.74 \\
\hline 5 & 6.69 & 3.70 & 13.69 & 7.33 & 15.83 & 8.49 & 16.06 & 8.62 \\
\hline 6 & 8.14 & 4.47 & 16.94 & 9.06 & 19.56 & 10.51 & 19.84 & 10.65 \\
\hline
\end{tabular}

Table 3. Simulation results for the generalized f-CDTM and the CDTM

\begin{tabular}{|c|c|c|c|c|c|c|c|c|}
\hline & \multicolumn{2}{|c|}{$\begin{array}{c}N_{1}=\infty, N_{2}=12 \\
\text { (gener. f-CDTM) }\end{array}$} & \multicolumn{2}{c|}{$\begin{array}{c}N_{1}=\infty, N_{2}=60 \\
\text { (gener. f-CDTM) }\end{array}$} & \multicolumn{2}{c|}{$\begin{array}{c}N_{1}=\infty, N_{2}=600 \\
\text { (gener. f-CDTM) }\end{array}$} & \multicolumn{2}{|c|}{$\begin{array}{c}N_{1}=N_{2}=\infty \\
\text { (CDTM) }\end{array}$} \\
\hline $\mathrm{P}$ & $B_{1 c_{2}}(\%)$ & $B_{2 c_{1}}(\%)$ & $B_{1 c_{2}}(\%)$ & $B_{2 c_{1}}(\%)$ & $B_{1 c_{2}}(\%)$ & $B_{2 c_{1}}(\%)$ & $B_{1 c_{2}}(\%)$ & $B_{2 c_{1}}(\%)$ \\
\hline 1 & $1.98 \pm 0.09$ & $0.97 \pm 0.05$ & $3.10 \pm 0.27$ & $1.86 \pm 0.04$ & $3.66 \pm 0.18$ & $2.20 \pm 0.11$ & $3.81 \pm 0.34$ & $2.25 \pm 0.18$ \\
\hline 2 & $2.66 \pm 0.15$ & $1.38 \pm 0.05$ & $4.34 \pm 0.11$ & $2.79 \pm 0.09$ & $5.22 \pm 0.17$ & $3.32 \pm 0.08$ & $5.29 \pm 0.38$ & $3.55 \pm 0.27$ \\
\hline 3 & $3.44 \pm 0.26$ & $1.82 \pm 0.07$ & $6.17 \pm 0.22$ & $3.95 \pm 0.08$ & $7.38 \pm 0.25$ & $4.60 \pm 0.17$ & $7.46 \pm 0.57$ & $4.87 \pm 0.15$ \\
\hline 4 & $4.35 \pm 0.17$ & $2.29 \pm 0.07$ & $8.34 \pm 0.37$ & $5.32 \pm 0.19$ & $9.66 \pm 0.40$ & $6.34 \pm 0.09$ & $9.80 \pm 0.55$ & $6.45 \pm 0.23$ \\
\hline 5 & $5.32 \pm 0.17$ & $2.85 \pm 0.04$ & $10.22 \pm 0.18$ & $6.94 \pm 0.22$ & $12.11 \pm 0.31$ & $8.23 \pm 0.15$ & $11.87 \pm 0.29$ & $8.61 \pm 0.67$ \\
\hline 6 & $6.49 \pm 0.23$ & $3.48 \pm 0.10$ & $12.66 \pm 0.29$ & $8.38 \pm 0.23$ & $14.86 \pm 0.40$ & $10.57 \pm 0.16$ & $15.44 \pm 0.45$ & $10.63 \pm 0.56$ \\
\hline
\end{tabular}

\section{Conclusion}

We've proved the f-CDTM and shown its performance together with the generalized f-CDTM, by comparing their (numerical) CBP results with simulation results.

\section{References}

1. J.S. Kaufman, "Blocking in a Shared Resource Environment", IEEE Trans. Commun. 29 (10) pp. 1474-1481, 1981.

2. J.W. Roberts, "A service system with heterogeneous user requirements", in: G. Pujolle (Ed.), Performance of Data Communications systems and their applications, North Holland, Amsterdam, pp.423-431,1981.

3. J.S. Kaufman, "Blocking in a Completely Shared Resource Environment With State Dependent Resource And Residency Requirements”, Proc. IEEE INFOCOM'92, pp. 22242232, 1992.

4. J.S. Kaufman, "Blocking with retrials in a completely shared resource environment", NorthHolland, Performance Evaluation, 15, pp. 99-113, 1992.

5. I. Moscholios, M. Logothetis and G. Kokkinakis, "Connection Dependent Threshold Model: A Generalization of the Erlang Multiple Rate Loss Model", Performance Evaluation, Vol.48, Issue 1-4, pp. 177-200, May 2002.

6. G. Stamatelos and J. Hayes, "Admission control techniques with application to broadband networks”, Comput. Commun.,Vol. 17, No. 9, pp. 663-673, September 1994. 